\title{
Italian National Data Bank of Stillbirth versus Sudden Infant Death Syndrome (SIDS) - A New Epidemiological and Pathological Perspective
}

Luigi Matturri ${ }^{1}$, Anna Maria Lavezzi ${ }^{1 *}$ and Giuseppe Del Corno ${ }^{2}$

1"Lino Rossi" Research Center for the study and prevention of sudden perinatal death and SIDS - Department of Surgical, Reconstructive and Diagnostic Sciences, University of Milan, Milan 20122, Italy

${ }^{2}$ Department of Experimental Medicine, University of Milan-Bicocca, Milan, Italy

\begin{abstract}
Aim: To contribute to the epidemiological evaluation of the risk factors underlying unexplained fetal death and SIDS, as imposed by the Italian Law 31/2006, and a more balanced assessment of the morphological substrates of these deaths.

Methods: Fifty nine sudden fetal deaths and 61 SIDS cases were compared to 120 matched controls randomly selected from live born child of mother living in the same catchment area, to assess if one or more exogenous variables could promote unexpected death. In-depth anatomo-pathological examinations were performed on the autonomic nervous and cardiac conduction systems in the dead victims.

Results: Prematurity and maternal smoking are determinant factors of infant mortality. In particular mothers smoking during pregnancy experienced a two-fold increase in risk of sudden fetal death and SIDS relative to mothers who never smoked. Besides, the localization and the nature of a variety of specific congenital abnormalities of the autonomic nervous system and of the cardiac conduction system were highlighted as morphological substrates of the pathophysiological mechanism of unexpected death.

Conclusion: Maternal smoking during pregnancy is associated with a significantly increased risk of unexpected fetal mortality and SIDS. Thus it is important to promote information campaigns to reduce tobacco use particularly in young woman.
\end{abstract}

Keywords: SIDS; Unexplained stillbirth; Risk factors; Data bank

Abbreviations: ANS: Autonomic Nervous System; AVN: Atrioventricular Node; CCS: Cardiac Conduction System; SAN: Sino-atrial Node; SIDS: Sudden Infant Death Syndrome

\section{Introduction}

As announced in a previous report [1], here we present a first analysis of all the information related to victims of sudden and unexplained fetal and infant death collected in the data bank established under the Italian Law 31/2006 "Regulations for Diagnostic Post Mortem Investigation in Victims of Sudden Infant Death Syndrome (SIDS) and Unexpected Fetal Death.” This law mandates in fact that infants within one year of life, and stillbirths after 22 weeks of gestation suddenly died without any apparent cause, must be submitted to diagnostic postmortem investigation and that the findings of the in-depth autopsy, the information about the pregnancy, fetal development and delivery, and, in the case of SIDS, the environmental and familial situation in which the death occurred, in addition to information related to risk factors, must be collected by the obstetrician gynecologist, neonatologist, pediatrician, and pathologist involved in the case and recorded in the registry of the data bank.

Until the promulgation of the Law, little effort was made to find the etiopathogenesis of these deaths and no database existed from which researchers were able to store autopsy results and all the information about the circumstantial, environmental, and familial situation within which the death occurred. Thus, basic information in this field was inadequate.

The purpose of this study was to analyze all the information included in the Italian data bank related to 59 unexpected fetal deaths and 61 SIDS victims. The anatomopathological protocol applied for the post-mortem investigation included in particular the study in serial sections of the autonomic nervous system and of the cardiac conduction system [2-4].

To evaluate if one or more of the variables is really involved in sudden fetal or infant death, the 120 cases of unexpected death were compared to 120 matched controls.

The analysis of the risk factors, particularly of those classified as "preventable" according to Guntheroth's criteria [5], performed in this case-control study will allow to update the population through public education and prevention programs aimed at decreasing the incidence of stillbirth and SIDS.

\section{Material and Methods}

The case-control study included 240 subjects, namely three groups of Caucasian infants: a SIDS cohort (61 cases), an unexplained fetal cohort (59 cases), and a control group (120 cases). The victims of sudden death were ascertained by the "Lino Rossi" Center of the Milan University during the years 2001-2010, prevalently in Lombardy

*Corresponding author: Anna Maria Lavezzi, "Lino Rossi" Research Center Department of Surgical, Reconstructive and Diagnostic Sciences, University of Milan, Via della Commenda 19, 20122 Milan, Italy, Tel: +39/02-50320821; Fax: +39/02-50320823; E-mail: anna.lavezzi@unimi.it

Received March 27, 2012; Accepted May 22, 2012; Published May 24, 2012

Citation: Matturri L, Lavezzi AM, Del Corno G (2012) Italian National Data Bank of Stillbirth versus Sudden Infant Death Syndrome (SIDS) - A New Epidemiological and Pathological Perspective. J Neonatal Biol 1:104. doi:10.4172/21670897.1000104

Copyright: @ 2012 Matturri L, et al. This is an open-access article distributed under the terms of the Creative Commons Attribution License, which permits unrestricted use, distribution, and reproduction in any medium, provided the original author and source are credited. 
region, according to the above-mentioned national Law 31. This law states in particular that all infants dying of suspected SIDS and all fetuses who died after the 22th week of gestation without any apparent cause, must undergo an in-depth anatomopathological examination, according to the guidelines developed in our Research Center [2,3].

Hereafter we briefly describe the anatomopathological protocol. More detailed information is available on our previous publications [2-4,6-9].

\section{Protocol for the central autonomic nervous system (ANS) examination}

The brainstem and cerebellum, where the main vital centers are located (cardiorespiratory, arousal, upper digestive tract, etc.). were processed and embedded in paraffin. Transverse serial sections were made at intervals of $30 \mathrm{~mm}$. For each level, twelve $5 \mathrm{~mm}$ sections were obtained, two of which were routinely stained for histological examination using alternately hematoxylin-eosin and Klüver-Barrera stains. The remaining sections were saved and stained as deemed necessary for further investigations.

The main nuclei were analyzed in serial sections of the brainstem, namely the parafacial nucleus, the locus coeruleus, the retrotrapezoid nucleus, the parabrachial/Kölliker-Fuse complex and the rostral raphe nuclei in the pons and mesencephalon; the hypoglossus, the dorsal motor vagal, the tractus solitarius, the ambiguus, the preBötzinger, the inferior olivary, the arcuate and the caudal raphe nuclei in the medulla oblongata.

In the cerebellum, the cortex layers (external granular layer, molecular layer, Purkinje cell layer and internal granular layer) and the medullary deep nuclei (the dentate nucleus, the fastigial nucleus, the globose nucleus and the emboliform nucleus) were examined.

\section{Protocol for the examination of the cardiac conduction system (CCS)}

Two blocks of heart were removed and paraffin embedded. Block 1 consisted of a portion of the right atrial wall including the Sino-Atrial Node (SAN). Block 2, consisting of the lower portion of the atrial septum, essentially contains the Atrio-Ventricular Node (AVN). The two blocks were cut serially at intervals of $40 \mu \mathrm{m}$ (levels). For each level two sections of $8 \mu \mathrm{m}$ were stained alternately with hematoxylineosin and trichromic Heidenhain (Azan). The small hearts from fetuses aged 22-35 gestational weeks were instead processed entire and serially cut perpendicularly to the interatrial and interventricular septa.

\section{Protocol for the examination of the arteries}

The fetal and infant arteries (coronary, internal and external carotid, Willis arteries and aorta) were excised transversely to the longitudinal axis into segments approximately $2-4 \mathrm{~mm}$ long, processed and serially cut. The sections are stained with hematoxylineosin, Azan for histological examination, Alcian blue at $\mathrm{pH} 0.5$ and 2.5 for acid mucopolysaccharides analysis, Hyaluronidase-Alcian blue for hyaluronic acids detection and Weigert for elastic fibers identification.

\section{Collection of risk factors information - Statistical analysis}

All information related to both "non-preventable" and "preventable" risk factors were collected and stored in the specific data bank according to the guidelines provided by the above mentioned Law.

\begin{tabular}{|l|l|l|l|}
\hline VARIABLE & $\begin{array}{l}\text { CASES } \\
(\mathrm{n}=120) \\
\%\end{array}$ & $\begin{array}{l}\text { CONTROLS } \\
(\mathrm{n}=120) \\
\%\end{array}$ & $\mathbf{p}$ \\
\hline $\begin{array}{l}\text { GESTATIONAL AGE } \\
<37 \text { weeks } \\
\geq 37 \text { weeks }\end{array}$ & $\begin{array}{l}31.7(23.5-40.8) \\
68.3(59.2-76.5)\end{array}$ & $\begin{array}{l}0.8(0.0-4.6) \\
99.2(95.4-100.0)\end{array}$ & $* *$ \\
\hline $\begin{array}{l}\text { PARITY } \\
1\end{array}$ & $46.7(37.5-56.0)$ & $51.7(42.4-60.9)$ & \\
$>1$ & $53.3(44.0-62.5)$ & $48.3(39.1-57.6)$ & \\
\hline $\begin{array}{l}\text { WEIGHT } \\
<1500\end{array}$ & $12.5(7.2-19.8)$ & 0.0 & $*$ \\
$\begin{array}{l}1500-2499 \\
\geq 2500\end{array}$ & $22.5(15.4-31.0)$ & $2.5(0.5-7.1)$ & $* *$ \\
\hline $\begin{array}{l}\text { MATERNAL SMOKING } \\
\text { no } \\
\text { yes }\end{array}$ & $65.0(55.8-73.5)$ & $97.5(92.9-99.5)$ & \\
\hline $\begin{array}{l}\text { MATERNAL ALCOHOL/DRUG/ } \\
\text { SEDATIVE DRUG USE }\end{array}$ & $71.7(62.7-79.5)$ & $85.0(77.3-90.9)$ & $*$ \\
\hline $\begin{array}{l}\text { no } \\
\text { yes }\end{array}$ & $90.8(84.2-95.3)$ & $96.7(91.7-99.1)$ & \\
\hline $\begin{array}{l}\text { FEEDING (only SIDS) } \\
\text { human milk } \\
\text { formula/mixed }\end{array}$ & $9.2(4.7-15.8)$ & $3.3(0.9-8.3)$ & \\
\hline
\end{tabular}

** $p<0.01 * p<0.05$

Table 1: Distribution of potential risk factors for cases and controls (95\% confidence interval)

The non-preventable considered variables were: sex, gestational age, infant birth weight, parity, maternal age. Preventable risk factors included: parental cigarette smoking, alcohol and drug abuse, formula feeding. In the parental smoking group, particular attention was given to the maternal habit.

For each case of sudden unexplained death, one control was randomly selected from live born child of mother living in the same catchment area of cases and who had normal deliveries. Controls were matched pair wise to the index case on sex and age of mother $( \pm 2$ years). Information on the mother's smoking status and alcohol and drug use during pregnancy were collected from interviews. Parity, gestational age and the infant's birth weight of cases and controls were obtained from the hospital record. Categories of infant variables are grouped according to WHO definitions [10].

Conditional multiple logistic regressions were used to assess the effects of maternal smoking and drinking on fetal and infant death, adjusting for the simultaneous effects of other variables.

Ethics - Ethics approval for this study was granted by the Italian Health's Ministry in accordance with the above-mentioned Italian Law no. 31. Parents of all subjects died of sudden death provided written informed consent to autopsy under protocols approved by the Milan University L. Rossi Research Center institutional review board.

\section{Results}

Among the 120 cases examined in the study, 59 were fetal deaths (27 females and 32 males) aged 20-41 gestational weeks (mean: 35, median: 37, mode: 39) and 61 SIDS (33 females and 27 males) aged 25110 post conception weeks (mean: 50 , median: 47 , mode: 51 ).

Table 1 displays a comparison of the variables considered in the study by cases and by controls. Case infants were more likely to be "premature", that is pre-term ( $31.7 \%$ vs. $0.8 \%)$, and low ( $22.5 \%$ vs. $2.5 \%)$ or very low (12.5\% vs. $0.0 \%)$ birth weight than controls. A significant higher proportion of mothers of cases $(28.3 \%)$ in comparison with 


\begin{tabular}{|l|l|l|l|l|}
\hline \multirow{2}{*}{ Risk Factor } & \multicolumn{4}{|c|}{ RR } \\
\cline { 2 - 5 } & \multicolumn{3}{|c|}{ Crude } & \multicolumn{2}{c|}{ Adjusted* $^{*}$} \\
\hline Smoke & 2.33 & $(1.14-4.85)$ & 2.26 & $(1.03-4.99)$ \\
\hline Alcohol/Drugs & 2.75 & $(0.81-10.22)$ & 1.45 & $(0.31-6.85)$ \\
\hline
\end{tabular}

* controlling for birth weight, parity and gestational age

Table 2: Results of multivariate logistic regression analysis of risk of unexplained perinatal deaths and SIDS (95\% confidence interval)

\begin{tabular}{|c|c|c|}
\hline PATHOLOGICAL RESULTS & $\begin{array}{l}\text { Unxplained perinatal death victims } \\
\qquad(\mathrm{n} .45)\end{array}$ & $\begin{array}{l}\text { SIDS } \\
(n .36)\end{array}$ \\
\hline $\begin{array}{c}\text { ANS ALTERATIONS } \\
\text { (hypoplasia/agenesis/delayed } \\
\text { maturation) }\end{array}$ & Total number of cases & $\begin{array}{c}\text { Total } \\
\text { number of } \\
\text { cases }\end{array}$ \\
\hline \multicolumn{3}{|l|}{ Brainstem } \\
\hline Arcuate nucleus & 20 & 15 \\
\hline Pre-Bötzinger complex & 7 & 3 \\
\hline Raphè nuclei & 12 & 5 \\
\hline Parafacial complex & $15^{*}$ & 1 \\
\hline $\begin{array}{l}\text { Parabrachial/Kölliker-Fuse } \\
\text { complex }\end{array}$ & $6^{* *}$ & - \\
\hline $\begin{array}{l}\text { Other nuclei (hypoglossal, } \\
\text { dorsal vagal, tractus solitarii, } \\
\text { inferior olivary) }\end{array}$ & 9 & 4 \\
\hline Cerebellar cortex & 4 & 5 \\
\hline $\begin{array}{l}\text { CCS ALTERATIONS } \\
\text { (resorptive } \\
\text { degeneration,Mahaim fibers, } \\
\text { cartilagineous metaplasia, } \\
\text { islands of conduction tissue in } \\
\text { the CF,..) }\end{array}$ & 15 & 7 \\
\hline $\begin{array}{l}\text { ATHEROSCLEROTIC } \\
\text { ALTERATIONS }\end{array}$ & 15 & 19 \\
\hline
\end{tabular}

ANS = autonomic nervous system

$\mathrm{CCS}=$ cardiac conductions system

${ }^{*} p<0.01$

**All victims of intra-partum death. (two-tailed Fisher's exact test: $p=0.031$ )

Individual victims may display any combination of these pathological alterations.

Table 3: Distribution of different histological alterations in the 81 cases with pathological diagnoses

mothers of controls (15.0\%) smoked during pregnancy; and there was a highly statistical difference of breastfeeding between SIDS cases (41.0\%) and their controls (71.2\%).

The results of multiple logistic regression analysis for maternal smoking and alcohol/drug use during pregnancy are presented in Table 2. As to the two maternal risk factors, the adjusted OR for the smokers was about 2.3 times greater than that for the non-smokers, but the OR associated with alcohol/drug use was substantially reduced after adjustment instead.

Only in 97 of the 120 cases we were able to perform an in-depth histological examination. In the other 23 cases the samples were inadequate (frequently due to maceration processes) for histological examination. Developmental alterations of both the autonomic nervous system and of the cardiac conduction system were detected in 81 cases. None alteration was highlighted in the remaining 16 cases.

A detailed report of all the pathologic results is represented in Table 3.

We can summarize the results as follows:

- Both unexplained perinatal death and SIDS share common congenital anomalies of the central ANS and CCS.
- The alterations of the brainstem and cerebellum vital centers are wider and more severe in fetal deaths as compared to SIDS. In fact, whereas in SIDS victims the more frequent observation is the hypoplasia of the arcuate nucleus, unexpected antepartum deaths are characterized by hypodevelopment of various structures: hypoplasia of the arcuate nucleus, of the parabrachial/Kölliker-Fuse complex, of the pre-Bötzinger complex, of the raphe nuclei and of the parafacial complex. In particular, this last lesion represents a marker of unexpected ante-partum death. In fact it is found exclusively, and with a high frequency (over $30 \%, \mathrm{p}<0.01$ ), in fetal deaths. The distinctive congenital anomaly that marks intra-partum death is the hypoplasia of the parabrachial/Kölliker-Fuse complex $(\mathrm{p}=0.031)$.

- Alterations of the CCS (resorptive degeneration, accessory AV pathways, mainly Mahaim fibers, cartilagineous metaplasia, etc.) are more frequent in all in stillbirths than in SIDS.

- Multifocal coronary early atherosclerotic lesions of varying entities were detected from the 30th gestational week particularly in the coronary and cerebral arteries, in the carotid and aorta. The alterations ranged from severe alteration of the cyto-architecture of the tunica media, forming perpendicularly oriented columns of smooth muscle cells infiltrating the intimae to focal plaques with mild myointimal thickening and also juvenile soft plaques reducing the arterial lumen.

\section{Discussion}

Only recently the interest of researchers has turned to an in-depth study of the ANS structures which modulate vital functions and whose anomalies may be the death mechanism substrate. The common denominator of these deaths is the absence of neurological and/or cardiac symptoms, usually associated with the presence of congenital abnormalities of both the ANS system, central and peripheral, which control breathing activity, and of cardio-vascular functions, as well as arousal, after birth.

The assessment of the knowledge deduced in this study from in-depth histological examination of the ANS and CCS in a wide sample of unexpected fetal deaths and SIDS victims, together with our previous studies [11-20], has led to the recognition of structural abnormalities, mostly congenital, that may plausibly account for a disruption of the neural network, triggering lethal reflexes. Thus, the pathogenesis of unexplained stillbirth and SIDS seems to be related to disorders in the control of the vital functions.

Our neuropathological investigations in particular have contributed to reveal the localization and the nature of a variety of congenital abnormalities of the ANS, particularly of the brainstem and cerebellum. From the overall analysis of the results we can say that in general the changes are more extensive, involving a greater number of vital centers, in stillbirths than in SIDS. However unexplained fetal death should not be regarded as distinct from the cot death, since all these deaths are now seen to be due to the expression of same congenital abnormalities of an integral part of the autonomic nervous system. Therefore, the definitions of these deaths, currently nosographically distinct, should be unified.

This new approach is particularly recommended to tackle the multifactorial, controversial substrates of these deaths and is prompting important debate, providing arguments for a broader discussion of the pathogenesis and nosology of this open problem. 
For each victim included in this case-control study, anamnestic and epidemiological data were collected in a specific questionnaire included in our protocol and inserted in the data bank. The variables commonly referred as risk factors for fetal/infant sudden and unexplained death were classified, according to Guntheroth's criteria [5], as "non-preventable" and "preventable".

Among the "non-preventable" risk factors we have to emphasize the "prematurity", a determinant factor that unfortunately is frequently neglected as cause of infant mortality. Concerning this, Sowards [21] presented an updated etiology-based cause classification system of infant deaths, which illustrates the potential underestimation of prematurity-related mortality in current vital statistics reports. In fact, sudden infant deaths are often assigned, according to the International Classification of Diseases [22], to code other than "disorders related to short gestation and low birth weight". In the present study we observed a statistically significant correlation between prematurity (gestational age $<37$ weeks and birth weight $<$ $1500 \mathrm{~g}$ ) and sudden death as compared with controls $(\mathrm{p}<0.01)$.

Among the "preventable" risk factors particular attention was given to parental cigarette smoking and in particular to the maternal habit in pregnancy. The statistical evaluation of the exogenous variables suggests that maternal smoking during pregnancy is associated with a significantly increased risk of unexpected fetal mortality and SIDS. Such an association persists after adjustment for potentially confounding factors by multivariate analysis. After controlling, the estimated OR for smoking in this study is generally consistent with earlier investigations as a risk factor both for stillbirth [23-30] and SIDS [31]. The major competing hypothesis for the smoking effect observed in this study is that smokers differs from non smokers in other behavioral characteristics (e.g. drinking, drug use, etc), but controlling for these variables did not reduce the OR for smoking. The mechanism by which smoking may cause adverse pregnancy outcomes remains controversial and a bulk of theories have been proposed in this regard [31]. Moreover tobacco exposure has been associated with lower levels of serum folate and red blood cell folate in pregnant women [32] likely due to an important gene environment interaction between methylenetetrahydrofolate reductase gene activity and the smoke.

In cases of maternal smoking in pregnancy, carbon monoxide, a gaseous combustion product of nicotine, may readily cross by passive diffusion into the placenta, where it binds to hemoglobin. Consequently, carboxyhemoglobin, that is present in the fetal compartment at concentrations that are generally 15\% higher than the maternal levels, inhibits the release of oxygen into fetal tissues, causing hypoxia and a consequent delayed maturation especially in the most susceptible organs, including the brain [33]. Besides, nicotine is one of the few lipid-soluble substances that can spread beyond the blood-brain barrier by concentration gradient, and act directly on the expression of genes involved in the developing brain control. Therefore, among the numerous compounds of cigarette smoke, carbon monoxide and nicotine, as suggested by Gressness et al. [34], could affect the fetal brain via indirect and/or direct actions. In both cases, smoke exposure in utero may strongly affect the brain development, not only giving rise to structural abnormalities of neuronal centers checking the vital functions, but also impairing central chemo-sensitivity and neurotransmitter systems.

Similarly, prenatal nicotine absorption could alter the expression of specific genes, such as the T-box transcription factors that are required in the early development of the cardiac conduction system [35], and cause fetal arrhythmia in utero [36].

Furthermore, smoking, by affecting fetal oxygenation, causes vasoconstriction and reduces the fetal blood flood [37,38], as well as inducing atherosclerosis $[8,9,39]$.

According to other studies [40-43] no evidence confirms the conclusion that there is a significant risk between maternal alcohol or drug consumption and unexplained fetal death or SIDS.

In conclusion, all the alterations detected in both ANS and CCS of victims of sudden death, responsible of serious vagal reflexes, can be interpreted as expressions of a disturbed development as pathophysiological response to a variety of mechanisms and, above all, to maternal cigarette smoking in pregnancy.

These results suggest that all pregnant women should be advised that smoking places their unborn children in serious danger of suffering a variety of developmental alterations of the vital functions that can be causes of stillbirth and neonatal death.

Efforts to reduce tobacco use in pregnancy should focus on preventing cigarette smoking among teenage girls. In fact most women begin smoking in their teens and later find it very difficult to quit, even during pregnancy. For this purpose Italian Law 31 requires to promote prevention campaigns in order to guarantee a correct information on the problematic of SIDS and fetal death without an apparent cause, and to achieve programs of continuous formation in medicine for the obstetrician, the gynecologist, the pediatrician, the neonatologist, the pathologist, and the nursing staff involved in each case.

\section{Funding}

This study was supported by the Italian Health's Ministry in accordance with the art.3 of the Law 31/2006 "Regulations for Diagnostic Post Mortem Investigation in Victims of Sudden Infant Death Syndrome (SIDS) and Unexpected Fetal Death."

\section{Conflict of Interest}

All Authors declare that they have no conflicts of interest.

\section{References}

1. Matturri L, Giuliani P, Mecchia D, Pizzo F, Lavezzi AM (2010) Italian national data bank of stillbirth versus SIDS. Public Health Rep 125: 516.

2. Matturri L, Ottaviani G, Alfonsi G, Crippa M, Rossi L, et al. (2004) Study of the brainstem, particularly the arcuate nucleus, in sudden infant death syndrome (SIDS) and sudden intrauterine unexplained death (SIUD). Am J Forensic Med Pathol 25: 44-48.

3. Matturri L, Ottaviani G, Lavezzi AM (2005) Techniques and criteria in pathologic and forensic-medical diagnostics in sudden unexpected infant and perinatal death. Am J Clin Pathol 124: 259-268.

4. Matturri L, Ottaviani G, Lavezzi AM (2008) Guidelines for neuropathologic diagnostics of perinatal unexpected loss and sudden infant death syndrome (SIDS): a technical protocol. Virchows Arch 452: 19-25.

5. Guntheroth W (1999) Causation of SIDS: a critical review. Proceedings of International Symposium on SIDS. Milan, Italy 53-60.

6. Rossi L, Matturri L (1995) Anatomohistological features of the hearth's conduction system and innervation in SIDS. In: Rognum TO (Ed.) Sudden Infant Death syndrome: New trends in the nineties. Oslo: Scandinavian University Press 207: 12

7. Rossi L (1990) The conduction system and its innervation. Proc Kon Ned Akad Wetensch 93: 491-499.

8. Matturri L, Lavezzi AM, Ottaviani G, Rossi L (2003) Intimal preatherosclerotic 
Citation: Matturri L, Lavezzi AM, Del Corno G (2012) Italian National Data Bank of Stillbirth versus Sudden Infant Death Syndrome (SIDS) - A New Epidemiological and Pathological Perspective. J Neonatal Biol 1:104. doi:10.4172/2167-0897.1000104

Page 5 of 5

thickening of the coronary arteries in human fetuses of smoker mothers. $J$ Thromb Haemost 1: 2234-2238.

9. Matturri L, Ottaviani G, Corti G, Lavezzi AM (2004) Pathogenesis of early atherosclerotic lesions in infants. Pathol Res Pract 200: 403-410.

10. World Health Organization (WHO) (2006) Neonatal and perinatal mortality: country, regional and global estimates. pp 1-75.

11. Matturri L, Biondo B, Mercurio P, Rossi L (2000) Severe hypoplasia of medullary arcuate nucleus: quantitative analysis in sudden infant death syndrome. Acta Neuropathol 99: 371-375

12. Matturri L, Minoli I, Lavezzi AM, Cappellini A, Ramos S, et al. (2002) Hypoplasia of medullary arcuate nucleus in unexpected late fetal death (stillborn infants): a pathologic study. Pediatrics 109: E43.

13. Lavezzi AM, Matturri L (2008) Functional neuroanatomy of the human preBötzinger complex with particular reference to sudden unexplained perinatal and infant death. Neuropathology 28: 10-16.

14. Lavezzi AM, Ottaviani G, Rossi L, Matturri L (2004) Cytoarchitectural organization of the parabrachial/Kölliker-Fuse complex in man. Brain Dev 26: 316-320.

15. Lavezzi AM, Ottaviani G, Ballabio GM, Rossi L, Matturri L (2004) Preliminary study on the cytoarchitecture of the human parabrachial/Kölliker-fuse complex with reference to sudden infant death syndrome and sudden intrauterine unexplained death. Pediatr Dev Pathol 7: 171-179.

16. Lavezzi AM, Matturri L (2008) Hypoplasia of the Parafacial/Facial Complex A Very Frequent Finding in Sudden Unexplained Fetal Death. Open Neurosci J 2: $1-5$.

17. Lavezzi AM, Ottaviani G, Mingrone R, Matturri L (2005) Analysis of the human locus coeruleus in perinatal and infant sudden unexplained deaths. Possible role of the cigarette smoking in the development of this nucleus. Brain Res Dev Brain Res 154: 71-80.

18. Lavezzi AM, Casale V, Oneda R, Weese-Mayer DE, Matturri L (2009) Sudden infant death syndrome and sudden intrauterine unexplained death: correlation between hypoplasia of raphé nuclei and serotonin transporter gene promoter polymorphism. Pediatr Res 66: 22-27.

19. Lavezzi AM, Ottaviani G, Matturri L (2004) Role of somatostatin and apoptosis in breathing control in sudden perinatal and infant unexplained death. Clin Neuropathol 23: 304-310.

20. Lavezzi AM, Ottaviani G, Mauri M, Matturri L (2006) Alterations of biological features of the cerebellum in sudden perinatal and infant death. Curr Mol Med 6: 429-435

21. Sowards KA (1999) What is the leading cause of infant mortality? A note on the interpretation of official statistics. Am J Public Health 89: 1752-1754.

22. International Classification of diseases (1980) Ninth Revision. Geneva Switzerland: World Health Organization.

23. Clausson B, Gardosi J, Francis A, Cnattingius S (2001) Perinatal outcome in SGA births defined by customised versus population-based birthweight standards. BJOG 108: 830-834.

24. Froen JF, Arnestad M, Frey K, Vege A, Saugstad OD, et al. (2001) Risk factors for sudden intrauterine unexplained death: epidemiologic characteristics of singleton cases in Oslo, Norway, 1986-1995. Am J Obstet Gynecol 184: 694 702 .

25. Fretts RC (2005) Etiology and prevention of stillbirth. Am J Obstet Gynecol 193: 1923-1935.

26. Smith GC, Fretts RC (2007) Stillbirth. Lancet 370: 1715-1725.

27. Salihu HM, Sharma PP, Getahun D, Hedayatzadeh M, Peters S, et al. (2008) Prenatal tobacco use and risk of stillbirth: A case-control and bidirectional case-crossover study. Nicotine Tob Res 10: 159-166.

28. Dodds L, King WD, Fell DB, Armson BA, Allen A, et al. (2006) Stillbirth risk factors according to timing of exposure. Ann Epidemiol 16: 607-613.

29. Little RE, Weinberg CR (1993) Risk factors for antepartum and intrapartum stillbirth. Am J Epidemiol 137: 1177-1189.

30. Walles B, Tyden T, Herbst A, Ljungblad U, Rydhstrom H (1994) Maternal health care program and markers for late fetal death. Acta Obst Gynecol Scand 73: 773-778.
31. Sullivan FM, Barlow SM (2001) Review of risk factors for Sudden Infant Death Syndrome. Paediatr Perinat Epidemiol 15: 144-200.

32. McDonald S D, Perkins SL, Jodouin CA, Walker MC (2002) Folate levels in pregnant women who smoke: an important gene/environment interaction. Am J Obstet Gynecol 187: 620-625.

33. Lambers DS, Clark KE (1996) The maternal and fetal physiologic effects of nicotine. Semin Perinatol 20: 115-126.

34. Gressens P, Laudenbach V, Marret S (2003) [Mechanisms of action of tobacco smoke on the developing brain.] J Gynecol Obstet Biol Reprod (Paris) 32(1 Suppl): S30-S32.

35. Yutzey KE (2008) Teed off: cardiac conduction system development requires T-box transcription factors. Circ Res 102: 1295-1297.

36. Feng Y, Caiping M, Li C, Can R, Feichao X, et al. (2010) Fetal and offspring arrhythmia following exposure to nicotine during pregnancy. J Appl Toxicol 30: 53-58.

37. Lindblad A, Marsal K, Andersson KE (1988) Effect of nicotine on human fetal blood flow. Obstet Gynecol 72: 371-382.

38. Bruner JP, Forouzan I (1991) Smoking and buccally administered nicotine. Acute effect on uterine and umbilical artery Doppler flow velocity waveforms. J Reprod Med 36: 435-440.

39. Grafe MR (1994) The correlation of prenatal brain damage with placenta pathology. J Neuropathol Exp Neurol 53: 407-415.

40. Schellscheidt J, Ott A, Jorch G (1997) Epidemiological features of sudden infant death after a German intervention campaign in 1992. Eur J Pediatr 156: $655-660$

41. Hoffmann HJ, Damus K, Hillman L, Kongrad E (1988) Risk factors for SIDS results of the National Institute of Child Health and Human Development SIDS Cooperative Epidemiological Study. Ann N Y Acad Sci 553: 13-30.

42. Stewart AJ, Williams SM, Mitchell EA, Taylor BJ, Ford RP, et al. (1995) Antenatal and intrapartum factors associated with sudden infant death syndrome in the New Zealand Cot Death Study. J Paediatr Child Health 31 473-478.

43. Blair PS, Fleming PJ, Bensley D, Smith I, Bacon C, et al. (1996) Smoking and the sudden infant death syndrome: results from 1993-5 case-control study for confidential inquiry into stillbirths and deaths in Infancy. Confidential Enquiry into Stillbirths and Deaths Regional Coordinators and Researchers. BMJ 313 195-198. 